\title{
Improving Memory after Interruption: Exploiting Soft Constraints and Manipulating Information Access Cost
}

\author{
Phillip L. Morgan, John Patrick, Samuel M. Waldron, Sophia L. King, and Tanya Patrick \\ Cardiff University
}

\begin{abstract}
Forgetting what one was doing prior to interruption is an everyday problem. The recent soft constraints hypothesis (Gray, Sims, Fu, \& Schoelles, 2006) emphasizes the strategic adaptation of information processing strategy to the task environment. It predicts that increasing information access cost (IAC: the time, and physical and mental effort involved in accessing information) encourages a more memoryintensive strategy. Like interruptions, access costs are also intrinsic to most work environments, such as when opening documents and e-mails. Three experiments investigated whether increasing IAC during a simple copying task can be an effective method for reducing forgetting following interruption. IAC was designated Low (all information permanently visible), Medium (a mouse movement to uncover target information), or High (an additional few seconds to uncover such information). Experiment 1 found that recall improved across all three levels of IAC. Subsequent experiments found that High IAC facilitated resumption after interruption, particularly when interruption occurred on half of all trials (Experiment 2), and improved prospective memory following two different interrupting tasks, even when one involved the disruptive effect of using the same type of resource as the primary task (Experiment 3 ). The improvement of memory after interruption with increased IAC supports the prediction of the soft constraints hypothesis. The main disadvantage of a high access cost was a reduction in speed of task completion. The practicality of manipulating IAC as a design method for inducing a memory-intensive strategy to protect against forgetting is discussed.
\end{abstract}

Keywords: information access cost, interruption, memory, soft constraints hypothesis

There are many opportunities to interrupt people in workplace environments with the proliferation of increasingly sophisticated communication technologies. This has spawned a surge in literature over the last two decades investigating the effect of interruptions on performance (e.g., Gillie \& Broadbent, 1989; Hodgetts \& Jones, 2006a, 2006b; McFarlane, 2002). The evidence is universally negative from studies in many different contexts, including the office environment (e.g., DeMarco \& Lister, 1999; Jackson,

Phillip L. Morgan, John Patrick, Samuel M. Waldron, Sophia L. King, and Tanya Patrick are at the School of Psychology, Cardiff University, United Kingdom.

This work was funded by the United Kingdom MoD's Defence Technology Centre: Data and Information Fusion (MIMEX Cluster Project, DTC112) awarded to the second author. The views and conclusions contained in this document are those of the authors and should not be interpreted as representing the official policies, either expressed or implied, of the United Kingdom Ministry of Defence, or the United Kingdom Government.

We thank Andrew Howes and Geoffrey Duggan for their contribution to some initial ideas in this area and Geoffrey Duggan for programming the Blocks World Task. We are also grateful to Ciara Malone for running some of the participants in Experiments 2 and 3, and Katharine Montague for helping with data coding in Experiments 2 and 3. This paper has been much improved by the suggestions from Wendy Rogers, Greg Trafton, and two anonymous reviewers.

Correspondence concerning this article should be addressed to John Patrick, School of Psychology Cardiff University, Cardiff CF10 3AT, United Kingdom. E-mail: patrickj@cardifff.ac.uk
Dawson, \& Wilson, 2002, 2003; O'Conaill \& Frohlich, 1995), the aircraft flightdeck (e.g., Dismukes, Young \& Sumwalt, 1998; Latorella, 1998; Loukopoulos, Dismukes, \& Barshi, 2001, 2003), medical settings (Coiera \& Tombs, 1998; Tucker \& Spear, 2006), and nuclear power plants (Griffon-Fouco \& Ghertman, 1984).

Consequently, research has focused on developing methods for mitigating the negative effects of interruption. The present paper has the same applied goal but is novel in using the soft constraints hypothesis (Gray \& Fu, 2004; Gray, Sims, Fu, \& Schoelles, 2006) to develop a method for reducing forgetting due to interruption. Essentially the soft constraints hypothesis posits that cognitive strategies are adapted to the constraints of the task environment by assessing their utility in terms of the amount of performance time saved. Thus it is possible to control the selection of a particular strategy by manipulating parameters in the task environment that make it either more or less beneficial in terms of temporal constraints. One such parameter is information access cost (IAC), which with a small increase (e.g., a mouse movement and/or a brief time delay), induces a more intensive memory strategy ( $\mathrm{Fu} \&$ Gray, 2000; Gray \& Fu, 2004; Gray et al., 2006; Waldron, Patrick, Morgan, \& King, 2007) with increased resilience to forgetting of task information (Waldron et al., 2007). Suffering information access costs are intrinsic to our everyday computer environments when we try to avoid information clutter and have to pay such costs in opening and reopening applications, emails, or documents. Costs include a mouse movement, key press, and a time delay in accessing information. In other situations an access cost is imposed by interface designers, for example, when it is desirable to try and ensure that the "terms" of an agreement have been read or the 
deletion of a file has been confirmed through the imposition of a 'tick box.' This paper adopts the theoretical framework of the soft constraints hypothesis to investigate whether the strategy change associated with paying such access costs can have functional value in protecting against forgetting due to interruption, and if so, what advantages and disadvantages accrue.

First, the negative effects of interruption are reviewed briefly, followed by an overview of existing methods for avoiding such effects. Finally, the soft constraints hypothesis is discussed together with the predicted effects of manipulating IAC and its potential as a novel method to protect against forgetting due to interruption.

\section{Negative Effects of Interruption}

A wealth of research has shown that if a task is interrupted, performance is impaired when returning to that task. Negative effects include forgetting to execute an action after interruption (e.g., Einstein, McDaniel, Williford, Pagan, \& Dismukes, 2003; McDaniel, Einstein, Graham, \& Rall, 2004), delays in resuming the interrupted task (e.g., Hodgetts \& Jones, 2006a, 2006b; Monk, Trafton, \& Boehm-Davis, 2008; Trafton, Altmann, Brock, \& Mintz, 2003), increased task completion time (Eyrolle \& Cellier, 2000; Paquiot, Eyrolle, \& Cellier, 1986), decreased task accuracy (Bainbridge, 1984; Flynn et al., 1999), and elevated levels of stress (Zijlstra, Roe, Leonora \& Krediet, 1999). The level of impairment can be further inflated when a task is interrupted during high memory load (e.g., Monk, Boehm-Davis, \& Trafton, 2002), when interruption occurs relatively infrequently (Monk, 2004), when the interrupting and interrupted tasks require similar processing resources (e.g., Czerwinski, Chrisman, \& Rudisill, 1991; Gillie \& Broadbent, 1989, although see Latorella, 1996, for a contrasting account), and when the interruption is immediate and unanticipated, as compared with when there is a delay prior to switching tasks (e.g., Hodgetts \& Jones, 2003; Trafton et al., 2003).

In this paper, we assess the impact of interruptions in routine copying based tasks applicable to office settings in which information has to be copied and pasted. A similar task occurs in military command and control settings in which planners have to reorganize resources at a display to achieve an optimal attack or defense formation. Indeed copying, moving, and organizing images and data to achieve a particular goal has become an increasingly common task, and there is plenty of evidence that interruptions are frequent in such settings. For example, it has been estimated that interruptions take up approximately 10 minutes of every working hour (O'Conaill \& Frolich, 1995), and that workers are interrupted quite frequently at around 73 times per day (Cubesmart Inc., 2002) receiving in the region of 23 e-mails in a mediumsize U.K. company (Burgess, Jackson, \& Edwards, 2005).

\section{Methods for Mitigating the Effect of Interruption}

There are three main methods for reducing the negative effects of interruption: coordinating interruptions with performance of the primary task (McFarlane, 2002; McFarlane \& Latorella, 2002); using reminder cues (e.g., Cutrell, Czerwinski, \& Horvitz, 2001; Czerwinski, Cutrell, \& Horvitz, 2000; Franke, Daniels, \& McFarlane, 2002; McDaniel et al., 2004); and manipulating the interruption lag (e.g., Altmann \& Trafton, 2002, 2004; Hodgetts \& Jones, 2006a; Trafton et al., 2003). Each of these methods is reviewed briefly.

Four methods have been proposed for coordinating interruptions with computer-based work activities (McFarlane, 2002). An immediate switch to an interrupting task causes the largest performance impairment (e.g., Hodgetts \& Jones, 2006a; Trafton et al., 2003), whereas the negotiated method is associated with better task performance because the user has some control over when to switch to the interrupting task (McFarlane, 2002). In the mediated method, the task switch is governed by some prior measurement of the user's interruptibility whereas in the scheduled method this switch is predetermined. However, both of the latter methods were not only associated with fewer improvements in performance than the negotiated method, but were also found to introduce subsidiary problems. For example, a mediated method not only requires some supervisory control (Kirlik, 1993), but also has difficulty in accurately predicting a user's workload. A scheduled method, by definition, is limited to situations where interruptions can be delayed and queued. Therefore, any one method is not a panacea (McFarlane, 2002; McFarlane \& Latorella, 2002) and its efficacy will be determined by the criteria of task performance, the nature of the task environment, and user preference (Katz, 1995).

A different approach is the use of cues (e.g., post-it notes, diaries, calendars) to remind ourselves of what we may otherwise forget. In an interruption context, some have reported advantages of reminder cues (e.g., McDaniel et al., 2004), especially when fast resumption of the interrupted task is important (e.g., Hodgetts \& Jones, 2006a), although the effectiveness of using a mouse cursor as a place reminder is limited to when the processing demands of the interrupted task are quite low (e.g., Cutrell et al., 2001; Czerwinski et al., 2000). Reminder cues are generally more effective when they are attention grabbing, especially when located among other potentially competing stimuli in the task environment (e.g., Trafton, Altmann, \& Brock, 2005; Trafton et al., 2003). Another effective reminder method that circumvents the issue of cue salience is to provide a summary or log of recent preinterruption activities after completion of the interrupting task (Cypher, 1986; Franke et al., 2002). However such a technique may be difficult to implement and can further delay resumption of the task.

When it is possible to delay switching to an interrupting task, a popular manipulation is to include an interruption lag, which is a time delay between interruption annunciation (e.g., the telephone ringing) and the initiation of the interrupting task (e.g., answering the telephone) during which a user can prepare to return to the task following the interruption (Altmann \& Trafton, 2002). According to the goal activation model (Altmann \& Trafton, 2002), this is believed to involve rehearsing the interrupted goal and associating it with a salient reminder (or priming) cue located externally in the task environment or internally. Identification of the cue after an interruption is argued to cause reactivation and retrieval of the suspended goal, leading to faster resumption of the interrupted task (Hodgetts \& Jones, 2003; Trafton et al., 2003). Nevertheless, in a fairly complex task, an 8-s lag improved resumption time (Trafton et al., 2003) although the time saved at resumption was less than the time imposed by the lag. However, if the cost of slower resumption outweighs that of delaying the switch to an interrupted task, then a carefully timed interruption lag has an important practical benefit. 


\section{Soft Constraints Hypothesis and Manipulating}

\section{Access Cost}

The soft constraints hypothesis Gray \& Fu, 2004; Gray et al., 2006), with its roots in the adaptive control of thought-rational (ACT-R) theory (Anderson et al., 2004; Anderson \& Lebiere, 1998) proposes that changes in strategy are sensitive to millisecond changes to information presented in the task environment (Ballard, Hayhoe, Pook, \& Rao, 1997; Gray \& Boehm-Davis, 2000). Hard constraints determine behavior that is, or is not possible, whereas soft constraints control what strategy is most likely to be deployed. Cost-benefit adjustments are made with soft constraints at the 1/3to 3-s level of interactive routines that allow less effective strategies to be displaced by more effective ones in response to changes in the cost of the task environment. Effectiveness is determined by minimizing time as a performance cost, even when saving milliseconds might involve relying less on perfect information "in-the world" and more on previously encoded chunks of imperfect information "in-the-head" (Gray \& Fu, 2004). When information is permanently available in the task environment, a perceptual display-based strategy is likely to dominate. However, as IAC increases, performing the task with a display-based strategy will be rendered too costly in terms of time, which increases the likelihood of employing a memory-based strategy to offset this cost.

In support of the soft constraints hypothesis, recent work has shown that small costs (e.g., a physical movement and/or seconds delay) in accessing information can cause major shifts in processing strategy, with people committing larger chunks of information to memory (e.g., Fu \& Gray, 2000; Gray \& Fu, 2004; Gray et al., 2006; Waldron et al., 2007). Fu \& Gray (2000) were first to use the Blocks World Task (BWT), a visuospatial copying task originally developed by Ballard, Hayhoe, and Pelz (1995), to study the effects of varying access costs. In their study, information was occluded by masks and the three access cost conditions associated with uncovering the masks consisted of: a keyboard press (Low Cost condition), a mouse movement (Control condition), or a mouse movement and a one second delay to uncover (High Cost condition). As IAC increased, participants relied less on the external display as a memory source and instead committed more information to memory, as demonstrated by less visits to the target window but more time per visit spent encoding block information. The effect of increasing IAC on encouraging a more memorybased strategy has also been found during VCR programming (Duggan \& Payne, 2001; Gray \& Fu, 2001), with the cause being attributed to temporal cost-benefit tradeoffs (Gray et al., 2006) rather than the more traditional view of cognition operating to preserve memory (e.g., Wilson, 2002).

The following three experiments investigated whether the soft constraints hypothesis could be exploited to improve memory after interruption by manipulating IAC. Using the BWT, Experiment 1 further investigated the effect of varying IAC on task strategy and recall, and Experiment 2 examined whether these effects protected against forgetting after interruption and if any such benefit is dependent on interruption frequency. In Experiment 3, the utility of IAC was examined with a direct measure of prospective memory within the context of different types of interruption.

\section{Experiment 1}

In order to support the potential role of IAC in protecting against forgetting during an interruption, Experiment 1 aimed to replicate the previously reported effects of IAC on task completion strategies (Fu \& Gray, 2000; Gray \& Fu, 2004; Gray et al., 2006; Waldron et al., 2007) and to further investigate its effect directly on recall that has only been examined by Waldron et al. (2007). However, in the Waldron et al. study, there was no difference in recall between Medium and High IAC conditions suggesting that the extra time delay of one second between them was unimportant. As this finding is important not only theoretically to the theory of soft constraints but practically to the design and predictions of subsequent experiments in this paper, Experiment 1 was designed to further investigate the effect of these levels of IAC on recall. The BWT was selected as an experimental task because it was used in the above studies and it bore similarity to some of the practical copying tasks discussed above. In addition, the number of blocks encoded at each target visit, under the control of the participant, offered the opportunity for strategy to vary between a minimal and substantial memory load.

\section{Method}

\section{Participants}

Forty-eight Cardiff University students participated in the study for payment and were randomly assigned to one of three conditions. There were 43 women and five men with an age range of 19 to 34 years $(M=20.94, S D=3.01)$. Statistical power was calculated using $G^{*}$ Power 3.01 software (Faul, Erdfelder, Lang, \& Buchner, 2007), such that the sample size for Experiment 1 and subsequent experiments was adequate to detect a medium effect size with power of .8 .

\section{Materials}

The experiment was programmed and mouse movements and key presses were recorded. In the BWT, participants were required to copy a pattern of colored blocks from a target window (top-left of Figure 1) into a workspace window (top-right), by dragging blocks from a resources window (bottom-left). Target and workspace windows were the same size, and 10 colored blocks and six empty spaces were within each $4 \times 4$ grid. No colors were used twice, and the empty spaces were always gray. A different target pattern was used for each of the $10 \mathrm{BWT}$ problems, and a stop trial button was available for participants to press when they felt they had copied the pattern correctly, although if this was pressed prematurely, participants continued until the pattern was correct.

\section{Design}

IAC was manipulated on three levels. In the Low condition, the contents of all windows were permanently visible, representing a "normal" task environment. In contrast, windows were covered by a gray mask in both the Medium and High IAC conditions, and could only be uncovered by moving the mouse cursor over the window, but masks would reappear when the mouse cursor was moved out of the window. There was an additional 1-s delay to uncover the target window in the High IAC condition. IAC was manipulated betweensubjects to negate possible contamination from asymmetric transfer 

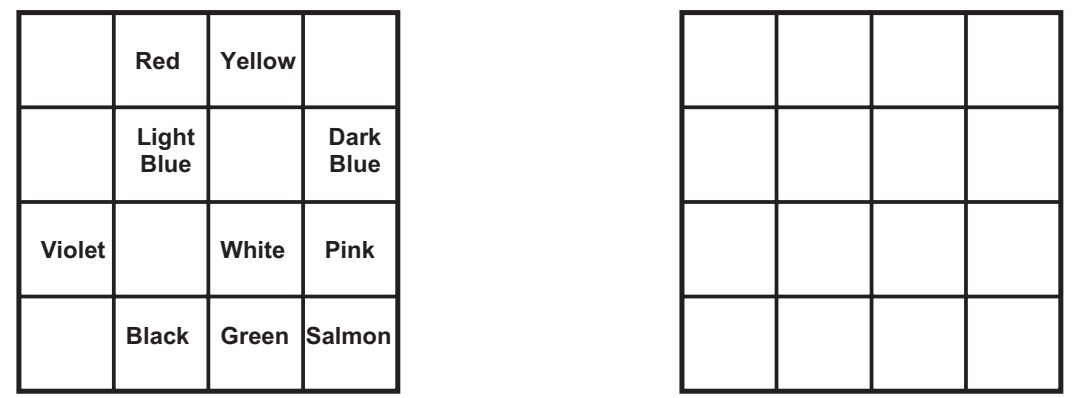

\begin{tabular}{|l|l|l|l|l|l|}
\hline Red & Violet & Yellow & Green & $\begin{array}{c}\text { Light } \\
\text { Blue }\end{array}$ & \\
\hline $\begin{array}{l}\text { Dark } \\
\text { Blue }\end{array}$ & Salmon & Pink & White & Black & \\
\hline
\end{tabular}

Stop Trial

Figure 1. Representation of the BWT interface for the Low IAC condition. Note. Target Window is top-left, Resources Window is bottom-left, and Workspace Window is top-right. Squares were presented as actual colors with no overlaying text as in Figure 1, and all blank squares were gray. (Also, actual colors were used in the task.)

(e.g., Poulton, 1982). There were 10 BWT problems with the fifth and tenth problems immediately followed by an unexpected recall test that required participants to recreate the target pattern while the goal state window was covered by a mask.

A number of dependent measures were used. First, and most important, the number of blocks correctly recalled on Trials 5 and 10 was compared among all conditions. Second, the number of blocks copied immediately after the first visit to the target window was compared between the Medium and High IAC conditions. Third, and to complement the second measure, a measure of the average time spent in the target window per visit was taken for the Medium and High IAC conditions. Throughout this paper, reference to a more intensive or stronger memory-based strategy between conditions indicates that more block information is encoded in the target window, which takes more time, and this is reflected in an increase in the number of blocks copied correctly into the workspace window. Finally, measures were taken to assess the impact of manipulating IAC on more global measures of task performance, including completion time and blocks incorrectly placed during trials.

\section{Procedure}

Participants were tested individually and were instructed how to perform the task. Following completion of two practice trials, participants completed 10 problems.

\section{Results}

First we consider the effects of IAC on recall followed by analyses of how any effects are due to the deployment of different processing strategies. Finally, the effects of varying IAC on the more global measures of speed and accuracy of performance are described. Throughout this paper, effect sizes are expressed as Cohen's $f$ (with an $f$ of .10 indicating a small effect size, .25 a medium effect size and .40 or above a large effect size; Cohen, 1988). Also, all outliers with z-scores higher than 3.29 were replaced with the grand mean (Field, 2005; Tabachnick \& Fidell, 2001).

\section{Recall}

Better recall was expected as IAC increased and this pattern was confirmed as a main effect of IAC, $F(2,45)=15.11, M S E=3.85$, $p<.001, f=.82$, (see Figure 2). Bonferroni post hoc tests found that participants in the High IAC condition recalled significantly

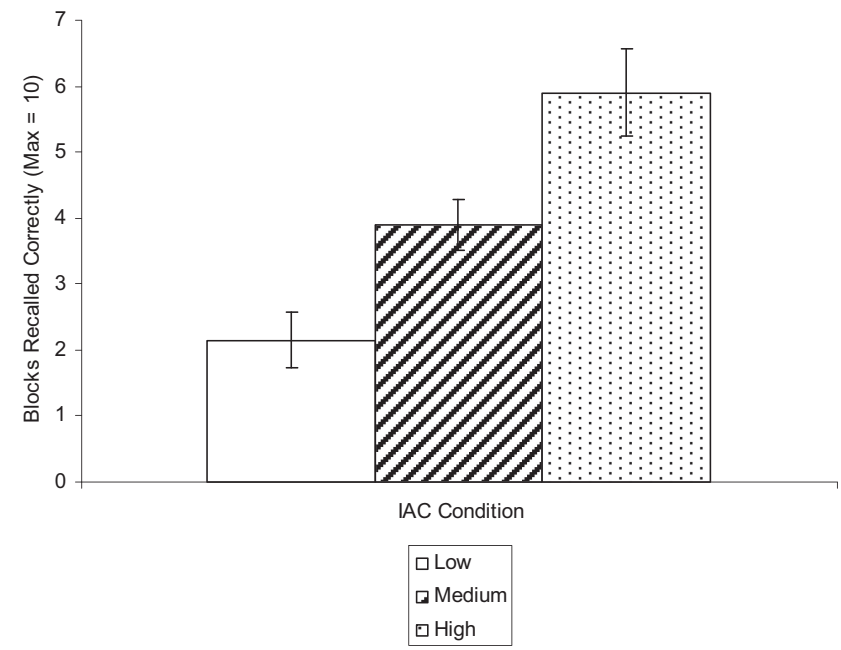

Figure 2. The effect of IAC on recall (Experiment 1). Error bars show standard error of mean. 
more blocks than those in both the Medium $(p<.05)$ and Low IAC conditions $(p<.001)$, and those in Medium recalled more than those in Low IAC $(p<.05)$.

\section{Task Strategies}

The more intensive memory-based strategy between Medium and High IAC would be indicated by more blocks copied correctly after viewing the goal-state for a longer time. (Directly comparable measures for Low IAC were not available.) Relevant data are presented in Table 1. First, participants copied more blocks immediately after the first visit to the target window in High than Medium IAC, $F(1,30)=$ $17.49, M S E=.69, p<.001, f=.76$. Second, participants spent more time encoding block information in the target window in High IAC than Medium IAC, $F(1,30)=16.15, M S E=.97, p<.001, f=.97$. These complementary measures point to the same conclusion that participants in the High IAC condition used a stronger memory-based strategy than those in Medium IAC.

\section{Global Task Performance}

By definition, High IAC comes at an extra time cost to overall task performance depending on the number of visits to the target window. Two measures of completion time were calculated: one of actual time that included lockout (High IAC) and the extra movement times (Medium and High IAC conditions), and another with these components eliminated, thus arguably making completion times of High and Medium IAC more comparable with Low IAC. Both measures are reported in Table 1, and participants in High IAC were slowest on both. Using the actual completion time (i.e., including lockout and extra movement times), IAC conditions differed, $F(2,45)=41.57, M S E=39.08, p<.001, f=1.36$, with participants in High taking longer to complete than those in both Medium and Low IAC $(p s<.001)$ and participants in Medium IAC taking longer than those in Low $(p<.001)$, see Table 1 . With both lockout and extra movement times eliminated (see Appendix) the same differences were found, albeit at slightly reduced significance levels ${ }^{1}$ ).

Table 1

Effect of IAC on Task Strategy and Global Task Performance (Experiment 1)

\begin{tabular}{llrr}
\hline & IAC & $M$ & $S D$ \\
\hline Strategy measures (per trial) & & & \\
$\quad$ Number of blocks copied correctly after first & High & 3.19 & .85 \\
$\quad$ target window visit & Med & 1.97 & .81 \\
Target window visit time (s) & High & 2.69 & 1.21 \\
& Med & 1.29 & .70 \\
& & & \\
Global performance measures (per trial) & High & 45.69 & 5.55 \\
Completion time (including 1-s lockout time & Med & 35.61 & 7.78 \\
$\quad$ in High IAC and extra mouse movement & Low & 25.54 & 5.09 \\
$\quad$ time to and from the target window in & & & \\
$\quad$ High and Medium IAC) (s) & High & 38.68 & 5.26 \\
Completion time (excluding 1-s lockout & Med & 32.86 & 7.78 \\
time in High IAC and extra mouse & & & \\
movement time to and from the target & & & \\
$\quad$ window in High and Medium IAC) (s) & High & 1.01 & .43 \\
Number of incorrectly placed blocks & Med & .67 & .47 \\
& Low & .51 & .29 \\
\hline
\end{tabular}

Consistent with a stronger memory-based strategy with High IAC, this condition was associated with participants making more errors (i.e., blocks copied to incorrect squares), $F(2,45)=6.52$, $M S E=.16, p<.01, f=.54$, although generally error rates were very low (see Table 1). Participants in the High IAC condition copied more blocks incorrectly than those in Low IAC $(p<.01)$ although participants in Medium IAC did not differ from those in either Low or High IAC.

\section{Discussion}

The increasingly intensive memory-based strategy as a function of increasing IAC resulted in improved recall among all IAC conditions. It was important that in contrast to Waldron et al. (2007), not only did the time delay of 1-s between Medium and High IAC result in increased recall, but so also did the extra mouse movement to open the target window between Medium and Low IAC. The effect of increasing access cost on task strategy is consistent with other studies (Fu \& Gray, 2000; Gray \& Fu, 2004).

These results support the soft constraints hypothesis (Gray et al., 2006) in demonstrating that a small change to the task environment can have significant consequences on strategy selection, such that when the cost of offloading memorial demands to the environment increases (i.e., having to suffer an extra mouse movement and/or a 1-s delay to inspect goal-state information), cognition will adapt to a more intensive memory-based strategy in order to save this local extra time cost. However this positive finding of improved recall with increased IAC in this copying task has to be weighed against the negative effects, largely on global completion time. Despite the saving in the local cost of inspecting target information with increasing IAC, global completion times increased correspondingly. Whether the tradeoff of better recall for slower speed is worthwhile will depend on the criteria of task performance and will vary between situations. These issues will be discussed fully at the end of this paper. However the goals of Experiment 1 to demonstrate that increased IAC directly improved recall for task relevant information were achieved.

\section{Experiment 2}

The main aim of Experiment 2 was to investigate whether the increasingly intensive memory-based strategy associated with increasing IAC, demonstrated in Experiment 1, could be employed to improve memory after interruption. Another aim was to investigate whether any such beneficial effect was dependent upon the frequency of interruption.

Predicting the effect of increasing frequency of interruption is not straightforward as there are few studies and both their empirical findings and explanations vary (Monk, 2004; Speier et al., 1999; Trafton et al., 2003). On the one hand, being interrupted more frequently can reduce the time to resume the task, whether frequency was defined as the number of times a task was interrupted (Monk, 2004) or as the number of trial blocks that were interrupted (Trafton et al., 2003). On the other hand, interrupting

\footnotetext{
${ }^{1}$ Completion times still differed among IAC conditions (see Table 1), $F(2,45)=18.24, M S E=38.03, p<.001, f=.90$, with High taking longer to complete than both Medium and Low IAC $(p s<.05)$ and Medium IAC taking longer than Low $(p<.01)$.
} 
complex tasks very frequently (e.g., 12 compared with 4 times per task) reduced accuracy and increased time to make decisions when resuming those tasks, the latter effect being attributed to an unsustainable increase in cognitive load rather than forgetting (Speier, Valacich, \& Vessey, 1999).

The soft constraints hypothesis offers a new theoretical perspective that can be used to explain and predict differing effects of not only interruptions but also increased frequency of interruption. As stated earlier, the soft constraints hypothesis proposes that the tradeoff in selection between a display- or memory-based strategy is determined by the associated costs in milliseconds for task performance. With respect to the memory side of the equation, Gray et al. (2006) proposed three factors that contribute to the utility and cost of a memory-based strategy: time spent encoding, retrieval time, and probability that the encoded item(s) can be recalled correctly when needed. Forgotten items represent a waste of both encoding time and retrieval time, plus the additional time required to recode and re-retrieve them. The nature and importance of these memory-related costs will depend upon the specific design of each interruption study. Interruptions can vary in duration between studies and therefore in how much they extend the period of time over which primary task items have to be recalled, during which they are subject to both decay and interference (e.g., Altmann \& Trafton, 2002). Also what task items have to be remembered can vary from a key press (e.g., McDaniel et al., 2004) to the attributes of both position and color of blocks in the BWT, which can impose a substantial memory load. The extent that encoding and rehearsal opportunities exist to compensate for a heavy memory load also varies between studies. According to the soft constraints hypothesis, the tradeoff in selection of strategy will depend upon weighing the overall effect of all these variables, specific to each study. In some situations, a memory-based strategy will cost little in terms of extra time and offer good utility and, in others, the investment in memory will not be justified by the probability of correct recall and associated time costs.

The effect of changing the frequency of interruption is simply to vary the number of occasions on which a strategy (e.g., memorybased) is experienced and assessed with respect to its utility, given values associated with a trial such as interruption duration, encoding opportunity, task item(s) decay function, and so forth. When it is advantageous and easy for task items to be recalled after an interruption with a memory-based strategy, increasing the frequency of interruption would be expected to have little effect on that strategy. However as recall probability declines with, for example, longer interruption duration, and encoding and retrieval times increase, then increased frequency of interruption will only serve to provide more evidence of the cost and lack of utility of an intensive memory-based strategy. We therefore do not expect such a strategy will be used under these circumstances with very high frequency of interruption.

Let us consider how these predictions relate to the BWT and Experiment 2. In High IAC (Experiment 1), the extra time cost of 1 -s spent uncovering the target window was sufficient to make it worthwhile for participants to spend more time encoding block information than those in Medium IAC. Similarly the extra mouse movement was sufficient to encourage participants in Medium IAC to encode more information than those in Low IAC. We therefore have observed an intensification of the memory-based strategy with increased IAC. If we introduce an interruption of a nontrivial duration, it will extend the period of time over which block information has to be retained and will increase encoding and retrieval costs, and the probability of it being forgotten (e.g., Altmann \& Trafton, 2002; Monk et al., 2008). This will be particularly important to High IAC that has extra lockout time and the strongest memory-based strategy. We predict that at infrequent and moderate frequencies of interruption in this condition, the increased memory costs and reduced recall probability may be tolerable and therefore will not effect a strategy change. It may even be the case that these levels of interruption strengthen the memory-based strategy if this tradeoff is still judged useful. However, with very frequent interruption, participants in the High IAC condition will experience almost continuously high memory costs and reduced recall probability that we predict will inhibit the more intensive memory-based strategy, associated with this condition in Experiment 1. Therefore Experiment 2 investigated whether increasing IAC can protect against forgetting due to interruption and whether the intensification of a memory-based strategy under High IAC will be inhibited with very frequent interruption.

\section{Method}

\section{Participants}

One-hundred and 44 Cardiff University students participated in the study for payment and were randomly assigned to one of 12 conditions. There were 123 women and 21 men with an age range of $18-39$ years $(M=20.32, S D=2.49)$. None had taken part in Experiment 1 .

\section{Materials}

There were 30 different BWT problems. Each interruption task involved solving a series of self-paced double-digit addition problems (e.g., $38+29=$ ?) over a period of 10 seconds. The interrupting tasks covered the whole screen. No feedback was given regarding performance on the interrupting tasks. All other features were the same as in Experiment 1.

\section{Design}

Both IAC and interruption frequency were manipulated between subjects. IAC was manipulated as in Experiment 1, except that the cost on the target window was increased from 1- to 2.5-s in High IAC with the aim of promoting an even stronger memory-based strategy. Low IAC was included in order to provide comparative data on recall and global measures of performance. Interruption frequency was manipulated with respect to how many problems were interrupted: none (control), 5 (infrequent), 15 (moderately frequent), or 25 (very frequent) of the 30 BWT problems. Problems were interrupted immediately after 2, 3, 4, 5, or 6 correct moves and these points of interruption were counterbalanced within each treatment combination.

To assess the effects of interruption, various resumption measures were calculated for the interrupted trials. These were the number of problems resumed without inspecting the target window, the number of blocks copied correctly in this situation, and the resumption lag. The same task strategy measures were used as in Experiment 1 and only one surprise recall test of the target 
Table 2

Effect of IAC and Interruption Frequency on Resumption Performance (Experiment 2)

\begin{tabular}{|c|c|c|c|c|c|c|c|}
\hline \multirow[b]{3}{*}{ Resumption measures } & \multirow[b]{3}{*}{ IAC } & \multicolumn{6}{|c|}{ Interruption frequency } \\
\hline & & \multicolumn{2}{|c|}{ Infrequent } & \multicolumn{2}{|c|}{$\begin{array}{l}\text { Moderately } \\
\text { frequent }\end{array}$} & \multicolumn{2}{|c|}{$\begin{array}{c}\text { Very } \\
\text { frequent }\end{array}$} \\
\hline & & $M$ & $S D$ & $M$ & $S D$ & $M$ & $S D$ \\
\hline \multirow{4}{*}{$\begin{array}{l}\text { Percentage of trials resumed without re-inspecting target } \\
\text { window } \\
\text { Number of blocks copied correctly without re-inspecting } \\
\text { target window }\end{array}$} & High & 21.67 & 32.41 & 53.17 & 28.39 & 25.75 & 14.43 \\
\hline & Med & 1.67 & 5.77 & 2.33 & 5.45 & 8.33 & 28.87 \\
\hline & High & 1.11 & .15 & 2.34 & .91 & 1.51 & .42 \\
\hline & Med & - & - & - & - & - & - \\
\hline \multirow[t]{3}{*}{ Resumption lag (s) } & High & 4.43 & 1.90 & 3.35 & 1.22 & 4.19 & 1.03 \\
\hline & Med & - & - & - & - & - & - \\
\hline & Low & 4.43 & .55 & 4.62 & .50 & 3.88 & .33 \\
\hline
\end{tabular}

Note. Participants in Medium IAC conditions resumed almost every trial by first revisiting the target window and therefore there was insufficient data to use on two of the above resumption measures for these conditions.

pattern occurred immediately after the last Trial 30 to avoid any adaptive change of strategy due to testing. Performance on interrupting tasks was assessed by the number of sums attempted and correct.

\section{Procedure}

This was the same as Experiment 1 except that one practice trial contained an example of the interrupting task, and participants then performed $30 \mathrm{BWT}$ tasks.

\section{Results}

Analyses of performance on the interrupting task were conducted in order to check for differences across IAC levels in the number of arithmetic problems attempted and correct. There were no significant differences with respect to IAC or its interaction with interruption frequency $(p s>.05)$ and therefore there was no evidence of differential rehearsal of the primary task varying with IAC.

First, the effects of IAC and interruption frequency on resumption after interruption are described and, second, the differing task strategies underpinning these effects are investigated together with associated recall differences. Finally, the effects of IAC on global task performance are described.

\section{Effects of IAC and Interruption Frequency on Resumption Performance}

Given the predicted memory effects with increased access cost, we were only interested in resumption of the primary task when no goal-state information was gleaned from reinspection of the target window after interruption. In this situation the resumption period was then defined as occurring between the end of the interrupting task and the first block copied to the workspace window. However, assessment of performance following interruption was complicated by the different resumption strategies adopted both between and within the Medium and High IAC conditions with respect to whether the target window was revisited immediately following interruption. Consequently, available data varied between treatment combinations.
Participants in the Medium IAC condition were unable to resume on almost every trial without reinspecting the target window, irrespective of frequency of interruption (see Table 2). In contrast, High IAC participants were able to resume on a number of trials without inspecting the target window (see Table 2), although a one-way analysis of variance (ANOVA) revealed that this varied with interruption frequency, $F(2,33)=$ 5.33, MSE $=.07, p<.01, f=.59$. The target window was viewed less by participants in High IAC before resumption with moderately frequent interruption than with infrequent and very frequent interruption ${ }^{2}(p s<.05)$. Furthermore, when High IAC participants resumed the task without inspecting the target window, they correctly copied an average of one or two blocks (see Table 2) although a one-way ANOVA revealed that again this varied with interruption frequency, $F(2,24)=6.62$, $M S E=.43, p<.01, f=.74$. Participants in the moderately frequent interruption condition under High IAC copied more blocks correctly than those in the other two frequency schedules $(p \mathrm{~s}<.05)$. These results indicate that, with increased IAC from Medium to High, direct resumption of the task occurs more frequently without the need for any review of the goal-state, and this effect is strongest in the High IAC, moderately frequent rather than very frequent interruption condition. This is consistent with the predicted reduction in the intensity of the memorybased strategy with very frequent interruption under High IAC.

Speed of resumption or resumption lag is traditionally an important measure in studies of interruption and the data for participants who resumed the task in High IAC without reinspecting the target window were compared against a baseline from Low IAC (see Table 2). The relatively small number of cases in which data were missing in the High IAC condition (six participants under infrequent and one participant under very frequent interruption) was dealt with by substitution with the overall experiment mean. A 2 (IAC: Low and High) $\times 3$ (interruption frequency: infrequent, moderately frequent, very frequent) ANOVA revealed no main

\footnotetext{
${ }^{2}$ A check was made to ensure that there were no differences in rehearsal opportunities across interruption frequencies in High IAC. There were no differences in either the number of sums attempted or correct $(p s>.05)$
} 
effects of IAC and interruption frequency on resumption lag although there was a significant interaction between these two factors, $F(2,66)=5.01, M S E=.79, p<.01, f=.39$, which was due to participants resuming faster under moderately frequent interruption with High IAC compared to those in the same frequency condition in Low IAC $(p<.01)$. Hence those participants in High IAC who resumed the task without inspecting the goalstate were not only as fast at resuming the task as those in the Low IAC condition, but were actually faster with moderately frequent interruption.

\section{Task Strategies and Recall}

It is important to confirm that the reduced susceptibility of High IAC to interruption, particularly at moderately frequent interruption, is due to changes in processing strategy, similar to those identified in Experiment 1. In order to avoid potential contamination from interruptions, data for High and Medium IAC were compared across the five noninterruption trials that were common to all interruption frequency conditions (Trials 1, 10, 17, 25, and 30). The same two task strategy measures were used, as in Experiment 1 , and the results were the same indicating that participants used a more memory-intensive strategy under High than Medium IAC (see Table 3). Participants in the High IAC condition copied more blocks correctly after the first visit to the target window, $F(1$, $88)=92.40, M S E=.83, p<.001, f=1.02$, and spent more encoding time in the target window per visit than Medium IAC, $F(1,88)=69.62, M S E=2.32, p<.001, f=.89$. In High IAC, more encoding time was spent in the target window per visit with moderately frequent than very frequent interruption $(p<.001)$.
A surprise recall test for the goal state on the last Trial 30 confirmed that participants recalled more as IAC increased (see Table 3), $F(2,132)=32.32, M S E=5.33, p<.001, f=.70$, and replicated the results of Experiment 1. High IAC participants recalled more than those in both Medium and Low IAC $(p<$ $.001)$, with Medium also better than Low IAC $(p<01)$. However, recall in High IAC was higher with moderately frequent than very frequent interruption $(p<.05)$, again supporting the prediction of a less intensive memory-based strategy with very than moderately frequent interruption under High IAC.

\section{Global Task Performance}

The positive effect of increasing IAC on mitigating some interruption effects has to be balanced against its negative effect on overall task performance, specifically on both speed and accuracy. Comparisons were made between IAC conditions across the same five noninterrupted trials common to all interruption frequency conditions with respect to both measures of task completion time (with the extra movement and lockout times both included or excluded) and the number of blocks incorrectly placed (see Table 3 ). Using actual task completion time, speed was slower with increasing IAC, $F(2,132)=162.4, M S E=63.15, p<.001, f=$ 1.56, with participants in the High IAC condition taking more time than those in Medium and Low IAC $(p s<.001)$. Also participants were slower to complete in High IAC with moderately frequent interruption compared to those experiencing very frequent interruption $(p<.05)$. The same completion time effects (and significance levels) occurred when lockout (High IAC) and extra movement times (Medium and High IAC) were excluded, even though

Table 3

Effect of IAC and Interruption Frequency on Task Strategy and Global Task Performance During Non-Interruption Trials (Experiment 2)

\begin{tabular}{|c|c|c|c|c|c|c|c|c|c|}
\hline & \multirow[b]{3}{*}{ IAC } & \multicolumn{8}{|c|}{ Interruption frequency } \\
\hline & & \multicolumn{2}{|c|}{ Control } & \multicolumn{2}{|c|}{ Infrequent } & \multicolumn{2}{|c|}{$\begin{array}{l}\text { Moderately } \\
\text { frequent }\end{array}$} & \multicolumn{2}{|c|}{$\begin{array}{c}\text { Very } \\
\text { frequent }\end{array}$} \\
\hline & & $M$ & $S D$ & $M$ & $S D$ & $M$ & $S D$ & $M$ & $S D$ \\
\hline \multicolumn{10}{|l|}{ Strategy measures (per trial) } \\
\hline \multirow{2}{*}{$\begin{array}{l}\text { Number of blocks copied correctly after first } \\
\text { target window visit }\end{array}$} & High & 3.55 & .98 & 3.67 & .81 & 4.63 & 1.56 & 3.72 & .81 \\
\hline & Med & 2.07 & .64 & 1.55 & .70 & 2.27 & .71 & 2.53 & .73 \\
\hline \multirow[t]{2}{*}{ Target window visit time (s) } & High & 3.78 & 1.64 & 3.68 & 1.73 & 5.19 & 3.30 & 2.60 & .59 \\
\hline & Med & 1.52 & .61 & .87 & .43 & 1.17 & .59 & 1.30 & .84 \\
\hline \multirow[t]{3}{*}{ Recall $(\max =10)$} & High & 4.5 & 2.71 & 5.08 & 3.23 & 6.75 & 2.70 & 4.00 & 3.28 \\
\hline & Med & 2.25 & 2.30 & 3.33 & 3.23 & 3.33 & 1.72 & 2.67 & 1.97 \\
\hline & Low & .58 & .79 & 2.58 & 1.56 & 1.25 & 1.06 & .92 & 1.38 \\
\hline \multicolumn{10}{|l|}{ Global measures (per trial) } \\
\hline \multirow{3}{*}{$\begin{array}{l}\text { Completion time (including } 2.5 \text {-s lockout time } \\
\text { in High IAC and extra mouse movement } \\
\text { time to and from the target window in High } \\
\text { and Medium IAC) (s) }\end{array}$} & High & 54.02 & 11.58 & 53.31 & 9.23 & 58.12 & 14.67 & 47.06 & 8.49 \\
\hline & Med & 37.68 & 4.85 & 36.18 & 5.74 & 35.07 & 10.39 & 32.56 & 5.75 \\
\hline & Low & 22.76 & 3.59 & 23.99 & 3.96 & 25.38 & 3.25 & 24.41 & 3.79 \\
\hline \multirow{2}{*}{$\begin{array}{l}\text { Completion time (excluding } 2.5 \text {-s lockout } \\
\text { time in High IAC and extra mouse } \\
\text { movement time to and from the target } \\
\text { window in High and Medium IAC) (s) }\end{array}$} & High & 42.20 & 10.29 & 42.68 & 8.75 & 48.22 & 14.33 & 36.01 & 5.81 \\
\hline & Med & 35.98 & 4.85 & 33.69 & 5.74 & 32.91 & 10.39 & 29.89 & 5.75 \\
\hline \multirow[t]{3}{*}{ Number of incorrectly placed blocks } & High & .88 & .99 & 1.22 & .74 & 2.68 & 2.53 & .67 & .58 \\
\hline & Med & .68 & .70 & .83 & .36 & .73 & .73 & .83 & .42 \\
\hline & Low & .20 & .19 & .30 & .31 & .32 & .26 & .45 & .66 \\
\hline
\end{tabular}


this reduced the completion times of these two conditions ${ }^{3}$ (see Table 3)

Errors in terms of blocks incorrectly placed per trial were generally low (see Table 3), but increased with increasing IAC, $F(2,132)=15.49, M S E=.85, p<.001, f=.46$. The effect of IAC on errors depended on interruption frequency, $F(6,132)=$ $4.08, M S E=.85, p<.01, f=.43$. More blocks under High IAC were incorrectly copied by participants with moderately frequent compared to very frequent interruption $(p<.001)$.

\section{Discussion}

The most important and novel finding of Experiment 2 is that increasing IAC can mitigate some of the negative effects of interruption. In order to secure this positive effect of IAC on resumption, access cost has to be more than a mouse movement and also involve a lockout cost of a few seconds on the target window. In this High IAC condition, more participants than under Medium IAC were able to resume the task without referring back to the goal-state and this effect was particularly strong with a moderately frequent interruption schedule. Furthermore, resumption lag was at its shortest in this condition; even shorter than all interruption frequencies under Low IAC where the target pattern was permanently visible and could have been relied upon as a resumption aid. The positive effect of High IAC on resumption was due to participants adopting a stronger memory-based strategy than those in Medium IAC for encoding the target pattern. Unequivocal and consistent evidence of this strategy was provided by results from a surprise recall test and a range of task strategy measures. According to the soft constraints hypothesis, a stronger memory-based strategy was adopted in High IAC because it minimized the need to inspect the target window and pay the penalty of the hard constraint of lockout time even though encoding and retrieval times increased together with increased probability of recall failure. Performance after interruption improved under High IAC as a result of this strategy.

However there is strong evidence that the frequency of interruption moderated the extent of the memory-based strategy under High IAC in line with our prediction. Strategy and recall measures indicated that the memory-based strategy was more intensive under High IAC at a moderate rather than a very frequent schedule of interruption. Consequently, resumption performance after interruption was best in this condition. We predicted that it would be difficult to sustain an intensive memory-based strategy under High IAC with a very high schedule of interruption. Essentially the nature of the tradeoff in strategy selection, postulated by the soft constraints hypothesis, changes with increased interruption frequency because of more evidence across more interruption trials of the extra memory time costs and the reduced probability of correct retrieval of block information. Interruptions imposed an extra 10-s period over which block information had to be retained on each interruption trial.

In order to understand how the above factors might have posed a too heavy memory burden under very frequent interruption, we analyzed the exact times over which block information had to be retained on both common noninterruption trials $(11,10,17,25$, \& $30)$ and common interruption trials $(2,9,16,21, \& 29)$ under High IAC. This was achieved by measuring the time between leaving the target window (having completed encoding block information) and leaving the workspace window (having completed retrieving and moving blocks ${ }^{4}$ ). On noninterruption trials, block information was retained for an average of 9.09-s although the effect of decay over this period (e.g., Brown, 1958; Peterson \& Peterson, 1959) could be offset to some extent by the increased encoding times that we observed. However, on interruption trials, block information was retained on average for 19.27-s and given a power decay function (e.g., Altmann \& Schunn, 2002; Altmann \& Trafton, 2002), compensating even for some of this through extra encoding time represents a considerable extra memory cost. From our empirical evidence of the effect of varying interruption frequency, when this cost is experienced on nearly every trial ( 25 out of 30 trials in the very frequent interruption schedule), selection of an intensive memory-based strategy is less attractive. In contrast, at a moderate frequency of interruption (interruption on half of the trials) such a strategy is still judged to be useful.

From a practical perspective the improved resumption performance with increasing IAC came at a cost to overall task performance in terms of decreased speed and reduced accuracy in this copying task. Whether such a sacrifice would ever be worthwhile in similar applied settings is discussed at the end of this paper.

\section{Experiment 3}

Experiment 3 had two aims. The primary aim was to further examine the protective effect of IAC on prospective memory postinterruption in a more direct and unambiguous fashion. In Experiment 2 it was possible for participants to choose whether or not to reinspect the target window following interruption and this resulted in different resumption strategies that varied with IAC. We wanted to have a cleaner and more comparable measure across all levels of IAC of how well future actions were remembered after interruption without any cues being available from the goal state Interruption experiments typically measure the effect of interruption by delay of resumption (e.g., Monk et al., 2008; Trafton et al., 2003) and completion time of the primary task (e.g., Eyrolle \& Cellier, 2000; Gillie \& Broadbent, 1989). Usually experiments do not measure directly prospective memory for future actions after interruption without the normal external cues being available in the task environment. Some studies that have examined prospective memory for a future action have used the delayed execution paradigm (Einstein et al., 2003; McDaniel et al., 2004), although memory is assessed by remembering to perform an action after resumption of the task. A novel aspect of Experiment 3 is that memory for future actions, planned before interruption when goal state information was available, was assessed without the goal state being available after interruption.

The second subsidiary aim was to test the protective effect of a memory-based strategy using High IAC by including a potentially more disruptive interrupting task than the arithmetic task used in Experiment 2. There is some debate in the literature concerning

\footnotetext{
${ }^{3}$ The same estimates of extra movement time in Medium and High IAC were used as described in the Appendix.

${ }^{4}$ Note that this will result in a slight overestimate in retention time because when the workspace window is vacated, block information will have been progressively retrieved and acted upon prior to this time.
} 
whether this might be achieved by selecting a very similar or dissimilar interrupting task in comparison to the primary task. On the one hand, there is evidence that interrupting tasks requiring very similar processing resources to the interrupted task are more disruptive to performance (Czerwinski et al., 1991; Edwards \& Gronlund, 1998; Gillie \& Broadbent, 1989). However, the apparent similarity effect in the last paper has since been criticized for being merely an effect of the complexity of the interrupting task (e.g., Latorella, 1996). A further set of experiments using a similar paradigm to Gillie and Broadbent captured an interruptionsimilarity effect although this effect could be abolished when the primary task was practiced in a fixed order before interruption (Edwards \& Gronlund, 1998). Attempting to categorize tasks as similar or dissimilar to the primary task is problematic as calibrating similarity is notoriously difficult and indeed this is a general criticism of the literature that has attempted to delineate the effect of supposedly similar and dissimilar interrupting tasks on primary task performance.

The theoretical literature concerning notions of transfer does predict what conditions will maximize negative transfer and therefore what should constitute the most disruptive interrupting task. These notions date back to the theory of identical elements proposed by Thorndike and Woodworth (1901), with subsequent modifications by Osgood (1949), Gick and Holyoak (1987), and Anderson (1987). These theories propose that processing new tasks and items that are identical to those already active in memory from the primary task causes most negative transfer. This is also consistent with the prediction from the search of associative memory model (Gillund \& Shiffrin, 1984; Raaijmakers \& Shiffrin, 1980), which posits that processed items are connected in memory by varying associative strengths, and those items that are highly similar will occupy proximal representational space. Greater interference occurs when trying to retrieve items due to representational proximity and the volume of items competing within this space. Consequently, in an attempt to maximize interference, we used the same interrupting task as the primary task that required identical encoding of the attributes of block color and position, albeit in a simpler version of the BWT.

\section{Method}

\section{Participants}

One hundred and eight Cardiff University students participated for payment and were randomly assigned to one of three IAC conditions. There were 98 women and 10 men, with an age range of $18-36(M=22.38, S D=3.66)$. None had taken part in Experiments 1 or 2 .

\section{Materials}

These were the same as those in Experiment 2, with the addition of an interrupting task that involved a $2 \times 2$ BWT problem containing four colored blocks.

\section{Design}

There were 35 BWT problems, 12 of which were used for two types of interruption and a control (no-interruption) condition positioned at one of two points during the solution sequence (after cor- rectly copying two or six blocks to the workspace window). Both interruption position and interruption types were counterbalanced. Given the design and resumption results in Experiment 2, interruption duration was reduced to 5-s and interruption frequency was a little less than one in two trials in order to try and encourage an intensive memory-based strategy that could be sustained with a potentially more disruptive interruption task. The new interrupting task was a $2 \times$ 2 BWT containing four colored blocks that was viewed for 2 -s, with the remaining 3-s available for recreating the target pattern in another $2 \times 2$ grid. The same arithmetic interrupting task was also used as in Experiment 2 in order to collect evidence of its effect under the revised prospective memory test situation, described previously. In the control condition, there was no interrupting task and no time delay. Prospective memory was tested immediately after interruption with participants attempting to complete as many more moves as they could with the target window occluded. The same three levels of IAC, as in Experiment 2, were manipulated between-subjects and the two types of interruption and the no-interruption control condition were manipulated within-subjects.

\section{Procedure}

This was the same as Experiments 1 and 2, except that participants in Experiment 3 received four practice trials followed by 35 BWT trials. One practice trial concerned completing the BWT normally without interruption and the other three practice trials involved the two different types of interruption plus the control noninterruption condition, all of these latter three conditions having a prospective memory test.

\section{Results}

Analyses of performance on the interrupting tasks were conducted in order to check for differences across IAC levels in the number of arithmetic problems attempted and correct for the arithmetic interrupting task and in the number of blocks copied and correct for the BWT interrupting task. There were no significant differences with respect to any measure $(p s>.05)$ and therefore there was no evidence of differential rehearsal opportunities of the primary task across levels of IAC.

First, results for prospective memory after interruption are described, followed by analysis of task strategies. Finally, overall speed and accuracy are described.

\section{Prospective Memory Following Interruption}

The efficacy of prospective memory was measured by the number of blocks copied correctly after interruption when the goal-state was no longer available (see Figure 3). Recall of future correct moves improved with increasing IAC, $F(2,105)=4.0$, $M S E=.79, p<.05, f=.28$, although this depended on the interruption condition as this factor interacted with IAC, $F(3.77$, 197.82 ) $=2.93, M S E=.39, p<.05, f=.24$, (a Huynh-Feldt correction was used due to a violation of sphericity). Participants in the control no-interruption condition recalled more than those in both interruption conditions in Medium and Low IAC ( $p$ s $<.01$ ), but this was not the case under High IAC (control vs. both BWT and mental arithmetic interruptions, $p \mathrm{~s}=.06$ ). Thus, both interruption tasks had the same negative effect on participants' pro- 


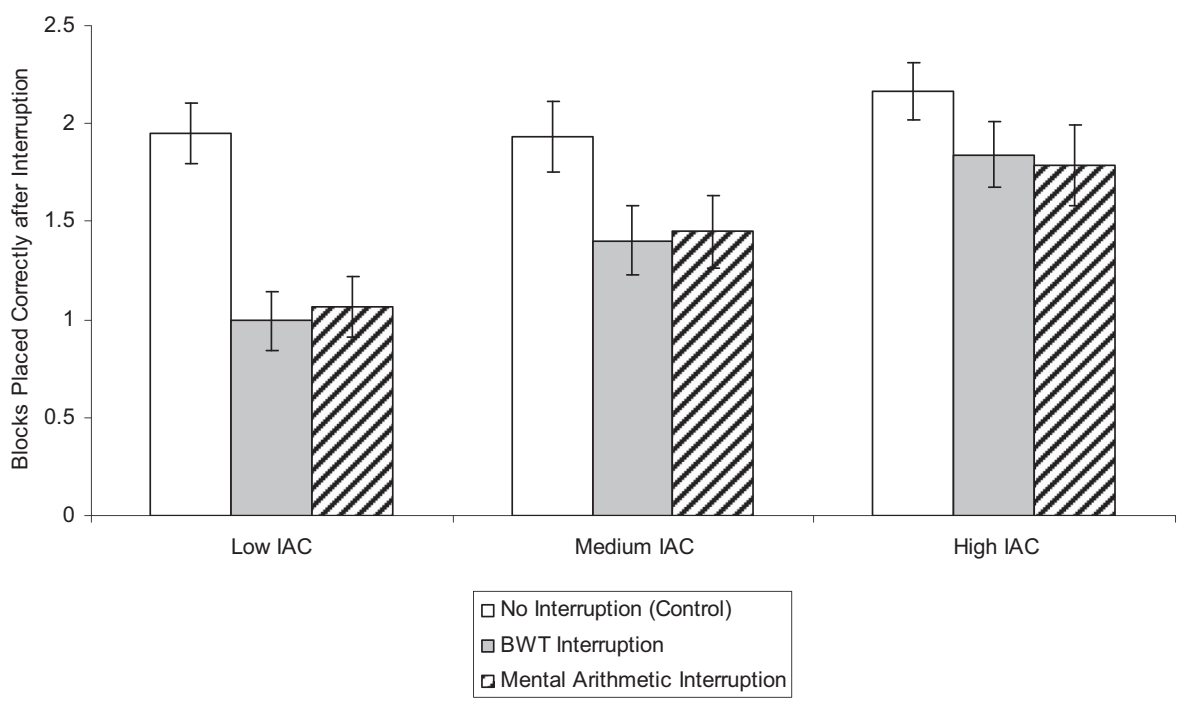

Figure 3. The effect of IAC and interruption type on prospective memory following interruption (Experiment 3). Error bars show standard error of mean.

spective memory in both Medium and Low IAC, although this negative effect was eliminated (statistically) for those in High IAC.

\section{Task Strategies}

Two of the same measures of task strategy from Experiments 1 and 2 were used to identify strategy differences between the Medium and High IAC conditions and the results were the same indicating a more memory-intensive strategy under High than Medium IAC (see Table 4). Participants in the High IAC condition copied more blocks correctly after the first visit to the target window, $F(1,70)=83.12, M S E=.67, p<.001, f=1.09$, and spent more encoding time in the target window per visit than those in Medium IAC, $F(1,70)=53.38, M S E=1.04, p<.001, f=.87$.

Table 4

Effect of IAC on Task Strategy and Global Task Performance During Non-Interruption Trials (Experiment 3)

\begin{tabular}{|c|c|c|c|}
\hline & IAC & $M$ & $S D$ \\
\hline \multicolumn{4}{|l|}{ Strategy measures (per trial) } \\
\hline \multirow{2}{*}{$\begin{array}{l}\text { Number of blocks copied correctly after first } \\
\text { target window visit }\end{array}$} & High & 3.84 & .75 \\
\hline & Med & 2.08 & .88 \\
\hline \multirow[t]{2}{*}{ Target window visit time (s) } & High & 3.71 & 1.97 \\
\hline & Med & 1.67 & .83 \\
\hline \multicolumn{4}{|l|}{ Global measures (per trial) } \\
\hline \multirow{3}{*}{$\begin{array}{l}\text { Completion time (including } 2.5 \text {-s lockout time } \\
\text { in High IAC and extra mouse movement } \\
\text { time to and from the target window in High } \\
\text { and Medium IAC) (s) }\end{array}$} & High & 51.62 & 6.67 \\
\hline & Med & 38.39 & 9.35 \\
\hline & Low & 25.73 & 5.02 \\
\hline \multirow{2}{*}{$\begin{array}{l}\text { Completion time (excluding } 2.5 \text {-s lockout } \\
\text { time in High IAC and extra mouse } \\
\text { movement time to and from the target } \\
\text { window in High and Medium IAC) (s) }\end{array}$} & High & 40.56 & 6.15 \\
\hline & Med & 36.42 & 9.35 \\
\hline \multirow[t]{3}{*}{ Number of incorrectly placed blocks } & High & 1.22 & .74 \\
\hline & Med & .75 & .57 \\
\hline & Low & .45 & .29 \\
\hline
\end{tabular}

\section{Global Task Performance}

Again two measures were used of task completion time (with the extra movement and lockout times both included or excluded $\left.{ }^{5}\right)$. As IAC increased, actual completion time increased (see Table 4), $F(1$, $105)=115.14, M S E=52.41, p<.001, f=1.48$, with participants in the High IAC condition taking longer to complete than those in both Medium and Low IAC, and participants in Medium IAC taking longer than those in Low $(p s<.001)$. With both lockout and extra movement times eliminated the same differences were found, albeit at slightly reduced significance level ${ }^{6}$. Error rates were again low but they increased with IAC, $F(1,105)=16.54, M S E=.32, p<.001$, $f=.56$, with participants in High making more errors than those in Medium IAC $(p<.01)$ and participants in Medium making more errors than those in Low IAC $(p<.001)$.

\section{Discussion}

The main results of Experiment 3 further confirm the capability of High IAC to mitigate some of the negative effects of interruption even when the interrupting task was designed to maximize interference with the primary task. Furthermore the results of Experiment 3 extend those of Experiment 2 because participants in High IAC were better able to resume from memory by demonstrating a similar improvement in this condition using a more direct measure of prospective memory following interruption. Again, there was unequivocal evidence of participants using a stronger memory-based strategy under High IAC that offered greater protection from both interrupting tasks than that used by

\footnotetext{
${ }^{5}$ The same estimates of extra movement time in Medium and High IAC were used as described in the Appendix.

${ }^{6}$ Completion times still differed among IAC conditions (see Table 1), $F(1,105)=42.01, M S E=50.20, p<.001, f=.89$, with High taking longer to complete than both Medium and Low IAC $(p s<.001)$ and Medium IAC taking longer than Low $(p<.05)$.
} 
participants in the Medium IAC condition. In High IAC the two interrupting tasks did not reduce postinterruption performance, as compared with no-interruption even though interruption involved switching to and from an interrupting task.

An initially surprising finding was the lack of difference in the number of blocks placed correctly among the IAC conditions with no-interruption (i.e., control conditions). One might have expected performance to improve with increasing IAC given that measures of strategy indicated clear differences between participants in High and Medium IAC. A likely explanation concerns the timing of the no-interruptions conditions, which were programmed to occur after making two or six correct moves in the workspace window, and how this related to the availability of the target pattern. In the no-interruption Low IAC condition, the target pattern only disappeared immediately prior to being asked to continue to make as many moves as could be remembered. In contrast, in the Medium and High IAC conditions, the target pattern will have been unavailable from the point at which a participant last moved the mouse cursor out of the target window. The duration of the unavailability of the target pattern prior to trying to remember moves would be determined by the time it took to move blocks in the workspace window to reach either of the two criteria of interruption that is, having positioned two or six blocks correctly in the workspace window. Thus participants in the Low IAC condition may be particularly advantaged in the no-interruption condition in comparison to those in both Medium and High IAC. The fact that there was no difference between the three nointerruption IAC conditions is testimony to the increased memorybased strategy used in the Medium and High IAC conditions that presumably compensated for the extra time over which future moves had to be remembered. Finally, and as in Experiments 1 and 2, High IAC came at a cost to overall task performance in terms of overall speed and accuracy, which is discussed below.

\section{General Discussion}

Three experiments involving a simple copying task found that making goal-state information harder to access in the task environment encouraged a memory-based strategy, which protected against some negative effects of interruption. Consequently this paper introduces IAC as a novel method for mitigating the negative effect of forgetting after interruption. A consistent finding across the three experiments was that small increases in IAC encouraged participants to use a cognitive strategy, more reliant on memory (see also Fu \& Gray, 2000; Gray \& Fu, 2004; Gray et al., 2006; Waldron et al., 2007). Consequently, recall for a previously completed task was superior with increasing access cost across all levels of IAC (Experiment 1), extending the findings of Waldron et al. (2007), and also when the copying task was interrupted (Experiment 2). High IAC was found to partly mitigate the negative effect of interruption on resumption and this was heightened when participants were interrupted with moderate frequency, that is, during half of the tasks (Experiment 2). Furthermore, High IAC protected against forgetting future actions even when the interruption required the same type of processing resource on a smaller scale (Experiment 3). Such access costs are relatively small and akin to those that naturally occur in computer-based environments and yet they produce a stronger memory-based strategy with accompanying medium to large effect sizes on postinterruption performance that are of potential practical importance.

The interruption studies in this paper provide a new test-bed for the recent soft constraints hypothesis (Gray \& Fu, 2004; Gray et al., 2006) and our results were consistent with predictions derived from it. In this theory, memory is viewed as adaptive, with soft constraints determining the utility and extent of a memory-based strategy depending on microscopic changes to the time costs of accessing information within the task environment. In the current studies, small increases in the cost of accessing information were shown to encourage an encoding strategy that was more memory dependent. Under High IAC, participants inspected the goal-state less, spent more time viewing it, and chunked more information during each visit. This intensive use of memory as a consequence of the hard constraint of lockout time under High IAC is consistent with the soft constraints hypothesis, but not with other views that assume cognition operates to conserve cognitive effort (e.g., Ballard et al., 1995; Cary \& Carlson, 2001; Wilson, 2002). The intensive memory-based strategy involves more effective encoding and storage strategies, such as chunking (Chase \& Ericsson, 1982; Chase \& Simon, 1973) and rehearsal (see Logie, 1995, for a review on rehearsal of visuospatial information). In contrast, less effortful display-based strategies are adopted when information is easily accessible within the task environment (e.g., Anderson \& Douglass, 2001; Duggan \& Payne, 2001; Waldron et al., 2007).

It may seem ironic that the tradeoff in strategy selection is judged at the local rather than the global level. In other words, although a stronger memory-based strategy may have minimized the local cost of paying lockout times, performance at the global level deteriorated. There are two important points. First, the participant had no control over the hard constraint of lockout time and therefore completion time would have probably increased further if a less memory-based processing strategy was adopted that required more inspections of the goal-state. Second, the fact that a local rational decision did not result in optimized global performance is not inconsistent with the soft constraints hypothesis (Gray et al., 2006), which states that task performance will only be optimized at a global level when all of the local tradeoffs complement each other in achieving this. There is evidence from many contexts that human behavior is driven by local rather than more distant or global payoffs and more weight is put on local events and their associated costs and benefits. This phenomenon has been termed "melioration" (Hernstein \& Vaughn, 1980) and more recently has been examined in terms of how it affects intertemporal choice even in economic domains (Lowenstein, Read, \& Baumeister, 2003).

It has to be acknowledged that the advantage of increased access cost of information to subsequent recall and postinterruption performance comes at a serious price with important practical ramifications, namely up to nearly double the time required to complete the task, and an increased error rate. The reduction in speed is mainly due to the increased time cost in accessing, encoding and retrieving information. Whether this price would be worth paying in applied situations will depend upon the criteria of task performance. If remembering some key information over a period of time, possibly involving interruptions, is of high importance then making information harder to access may be justifiable. On the other hand, if speed is important then it is unlikely that any increase in information access cost could be justified as, for example, in dynamic safety-critical situations in which a fast 
response is required. There are three caveats to add to this argument concerning whether the performance tradeoff with increased IAC could be beneficial. First, it is not suggested that all information within an interface should be made more difficult to access but only that associated with a variable whose value is of enduring significance. Second, it may be possible to make critical information harder to access some but not all of the time and still reap the benefits while increasing speed of performance. This is a topic for further research. Third, the benefit of IAC may be greater in more complex problem solving tasks in which there is a hierarchical goal structure as opposed to the flat goal structure of the copying tasks used in the current studies. If this were the case, then IAC may offer a more attractive solution to a broader range of task situations.

As highlighted at the beginning of this paper, the types of access cost utilized within the current experiments are akin to those regularly experienced in applied computing environments (e.g., working with overlapping windows, documents minimized to the taskbar etc.). However, even though such access costs occur naturally within computer environments, deliberately manipulating them may seem to contradict the principles of cognitive engineering. Generally cognitive engineering approaches strive to provide the user with immediately available relevant information that takes advantage of human perception and psychomotor abilities (e.g., Rasmussen \& Vicente, 1989; Woods, Wise, \& Hanes, 1981). For example, ecological interface design proposes that complex relationships between variables should be made directly accessible to operators in a manner that allows effortless extraction of information from the interface (Vicente \& Rasmussen, 1992). Also more recent engineering techniques such as information fusion (e.g., Dasarathy, 2001) make an even greater array of information available to the operator as quickly as possible in intelligent displays on the assumption that this is beneficial.

Shortcomings of this cognitive engineering approach have been well documented in the automation literature, (see Parasuraman \& Riley, 1997, for a review), with negative effects including the lack of system transparency, and the failure to keep the operator in the loop (e.g., Bainbridge, 1987). Similarly, making information highly accessible via the process of fusion may increase an operator's reliance upon the external display, and thus reduce the extent to which the displayed information is processed and committed to internal memory. A study by Waldron, Patrick, Duggan, Banbury, and Howes (2008) using a flight simulation found that providing location information on a temporary rather than permanent schedule improved its recall through a stronger memory-based strategy. These examples emphasize that there are exceptions to the common assumption that immediately accessible information is always preferable. This is consistent with our findings that manipulating IAC is another technique for effecting a more intensive memory-based strategy, the consequences of which may be desirable in some task situations, such as when key information has to recalled after interruption.

We need to acknowledge some limitations to our current studies. First, our results and associated interpretations were derived from use of the BWT and are therefore limited to copying type tasks without a hierarchical goal structure. Second, we have labeled three levels of IAC as Low, Medium, and High although IAC was not measured on a continuous scale and was a heterogeneous variable comprising not only time but also physical and mental effort. These factors were not separated in the present studies, although Gray et al. (2006) have attempted to delineate among them, concluding that their effect on time is the overriding factor. We would agree, given the many differences between the Medium and High IAC conditions, which only differed with respect to a time delay. Third, because eyetracking was not used, there were no comparable task strategy data available for the Low IAC conditions. However, our previous work using the BWT reported eyetracker data that did support the interpretation of a display-based task strategy under Low IAC (Waldron et al., 2007). Fourth, only one memory test was used in Experiment 2 and therefore we have no data on any trend over trials and also the recall data may have been affected by participants' surprise at this unheralded test. On the other hand, we wanted to collect evidence concerning recall that was not a strategic reflection of the knowledge of being tested. Finally, our demonstration of manipulating IAC as a possible method to mitigate some interruption effects is based only on one visuospatial copying task and a restricted range of parameters concerning interruption.

In conclusion, our studies address both theory and practice. The negative effects of interruption have received much recent attention in workplace settings (e.g., Milewski, 2006; Russell, Purvis, \& Banks, 2007; Tucker \& Spear, 2006), and in the laboratory (e.g., Hodgetts \& Jones, 2006a; McDaniel et al., 2004). Our studies exploited the theoretical framework of the soft constraints hypothesis (Gray et al., 2006) to develop a new means of mitigating some of the negative effect of forgetting following interruption by encouraging a more memory-based strategy with increased information access cost. In practice, our studies are relevant to displaybased copying tasks (e.g., office-based, military command and control), particularly when important information has to be retained, possibly because it is no longer available or has to be acted upon in a timely manner without having time to search again for an information source. Such a situation is relevant not only to everyday computer-based work situations but also safety-critical environments in which incidents occur because of a failure to recall critical task information after interruption, sometimes with devastating consequences (e.g., a fatal aircraft crash: NTSB, 1988). Future research should compare the utility of manipulating information access cost with other methods for alleviating interruption effects, using a wider range of tasks and applied settings.

\section{References}

Altmann, E. M., \& Schunn, C. D. (2002). Integrating decay and interference: A new look at an old interaction. Proceedings of the 24th annual meeting of the Cognitive Science Society (pp. 65-70). Hillsdale, NJ: Erlbaum.

Altmann, E. M., \& Trafton, G. J. (2002). Memory for goals: An activationbased model. Cognitive Science, 26, 39-83.

Altmann, E. M., \& Trafton, G. J. (2004). Task Interruption: Resumption lag and the role of cues. Proceedings of the 26th annual meeting of the Cognitive Science Society (pp. 42-47). Hillsdale, NJ: Erlbaum.

Anderson, J. R. (1987). Skill acquisition: Compilation of weak-method problem solutions. Psychological Review, 94, 192-210.

Anderson, J. R., Bothell, D., Byrne, M. D., Douglass, S., Lebiere, C., \& Qin, Y. (2004). An integrated theory of the mind. Psychological Review, 111, 1036-1360.

Anderson, J. R., \& Douglass, S. (2001). Tower of Hanoi: Evidence for the cost of goal retrieval. Journal of Experimental Psychology: Learning, Memory, and Cognition, 27, 1331-1346.

Anderson, J. R., \& Lebiere, C. (1998). The atomic components of thought. Mahwah, NJ: Erlbaum.

Bainbridge, L. (1984). Analysis of verbal protocols from a process control 
task. In E. Edwards \& F. P. Lees (Eds.), The human operator in process control (pp. 146-158). London: Taylor and Francis.

Bainbridge, L. (1987). Ironies of automation. In J. Rasmussen, K. Duncan, \& J. Leplat (Eds.), New technology and human error (pp. 271-283). New York: Wiley.

Ballard, D. H., Hayhoe, M. M., \& Pelz, J. B. (1995). Memory representations in natural tasks. Journal of Cognitive Neuroscience, 7, 66-80.

Ballard, D. H., Hayhoe, M. M., Pook, P. K., \& Rao, R. P. N. (1997). Deictic codes for the embodiment of cognition. Behavioral and Brain Sciences, 20, 723-767.

Brown, J. (1958). Some tests of the decay theory of immediate memory. Quarterly Journal of Experimental Psychology, 10, 12-21.

Burgess, A., Jackson, T. W., \& Edwards, J. (2005). Optimising the email communication environment. Managing modern organizations with information technology: IRMA 2005 proceedings. Hershey: IGP.

Card, S. K., English, W. K., \& Burr, B. J. (1978). Evaluation of mouse, rate-controlled isometric joystick, step keys and text keys for text selection on a CRT. Ergonomics, 21, 601-613.

Cary, M., \& Carlson, R. A. (2001). Distributing working memory resources during problem solving. Journal of Experimental Psychology: Learning, Memory, and Cognition, 27, 836-848.

Chase, W. G., \& Ericsson, K. A. (1982). Skill and working memory. In G. H. Bower (Ed.), The psychology of learning and motivation (Vol. 16, pp. 1-58). New York: Academic Press.

Chase, W. G., \& Simon, H. A. (1973). The mind's eye in chess. In W. G. Chase (Ed.), Visual information processing (pp. 215-281). New York: Academic Press.

Cohen, J. (1988). Statistical power analysis for the behavioural sciences (2nd ed.). Hillsdale, NJ: Erlbaum.

Coiera, E., \& Tombs, V. (1998). Communication behaviours in a hospital setting: An observational study. British Medical Journal, 316, 673-676.

Cubesmart Inc. (2002). Social interruption and the loss of productivity. Retrieved from: http://www.interruptions.net/literature/CubeSmartproductivity-wp1.pdf

Cutrell, E., Czerwinski, M., \& Horvitz, E. (2001). Notification, disruption and memory: Effects of messaging interruptions on memory and performance. In M. Hirose (Ed.), Human-computer interaction - INTERACT 2001 (pp. 263-269). IOS Press, IFIP.

Cypher, A. (1986). The structure of user's activities. In D. A. Norman \& S. W. Draper (Eds.), User centred system design (pp. 243-263). Hillsdale, NJ: Erlbaum.

Czerwinski, M., Chrisman, S. E., \& Rudisill, M. (1991). Interruptions in multitasking situations: The effects of similarity and warning (Tech. Rep. No. JSC-24757). Houston, TX: National Aeronautics and Space Administration (NASA).

Czerwinski, M., Cutrell, E., \& Horvitz, E. (2000). Instant messaging and interruption: Influence of task type on performance. In C. Paris, N. Ozkan, S. Howard, \& S. Lu. (Eds.), OZCHI 2000 conference proceedings (pp. 356-361). Sydney, Australia.

Dasarathy, B. V. (2001). Information Fusion - what, where, why, when, and how? Information Fusion, 2, 75-76.

DeMarco, T., \& Lister, T. (1999). Peopleware: Productive projects and teams (2nd ed.). New York: Dorset House.

Dismukes, K., Young, K., \& Sumwalt, R. (1998). Cockpit interruptions and distractions: Effective management requires a careful balancing act. ASRS Directline, 10, 4-9.

Duggan, G. B., \& Payne, S. J. (2001). Interleaving reading and acting while following procedural instructions. Journal of Experimental Psychology: Applied, 7, 297-307.

Edwards, M. B., \& Gronlund, S. D. (1998). Task interruption and its effects on memory. Memory, 6, 665-687.

Einstein, G. O., McDaniel, M. A., Williford, C. L., Pagan, J. L., \& Dismukes, R. K. (2003). Forgetting of intentions in demanding situations is rapid. Journal of Experimental Psychology: Applied, 9, 147-162.
Eyrolle, H., \& Cellier, J. M. (2000). Some effects of interruptions in work activity: Field and laboratory results. Applied Ergonomics, 31, 537-543.

Faul, F., Erdfelder, E., Lang, A.-G., \& Buchner, A. (2007). G*Power 3: A flexible statistical power analysis program for the social, behavioral, and biomedical sciences. Behavioural Research Methods, 39, 175-191.

Field, A. (2005). Discovering statistics using SPSS (2nd ed.). Sage: London.

Flynn, E. A., Barker, K. N., Gibson, J. T., Pearson, R. E., Berger, B. A., \& Smith, L. A. (1999). Impact of interruptions and distractions on dispensing errors in an ambulatory care pharmacy. American Journal of Health System Pharmacy, 56, 1319-1325.

Franke, J., Daniels, J., \& McFarlane, D. (2002). Recovering context after interruption. In W. Gray \& C. Schunn (Eds.), Proceedings of the 24th annual meeting of the Cognitive Science Society (pp. 310-315). Hillsdale, NJ: Erlbaum.

Fu, W.-T., \& Gray, W. D. (2000). Memory versus perceptual-motor tradeoffs in a blocks world task. Proceedings of the 22nd annual conference of the Cognitive Science Society (pp. 154-159). Hillsdale, NJ: Erlbaum.

Gick, M. L., \& Holyoak, K. J. (1987). The cognitive basis of knowledge transfer. In S. M. Cornier \& J. D. Hagman (Eds.), Transfer of learning: Contemporary research and applications (pp. 9-47). San Diego: Academic Press.

Gillie, T., \& Broadbent, D. (1989). What makes interruptions disruptive? A study of length, similarity, and complexity. Psychological Research, 50, 243-250.

Gillund, G., \& Shiffrin, R. M. (1984). A retrieval model for both recognition and recall. Psychological Review, 91, 1-65.

Gray, W. D., \& Boehm-Davis, D. A. (2000). Milliseconds matter: An introduction to microstrategies and their use in describing and predicting interactive behavior. Journal of Experimental Psychology: Applied, 6, 322-335.

Gray, W. D., \& Fu, W.-T. (2001). Ignoring perfect knowledge in-the-world for imperfect knowledge in-the-head: Implications of rational analysis for interface design. CHI Letters, 3, 112-119.

Gray, W. D., \& Fu, W.-T. (2004). Soft constraints in interactive behaviour: The case of ignoring perfect knowledge in-the-world for imperfect knowledge in-the-head. Cognitive Science, 28, 359-383.

Gray, W. D., Sims, C. R., Fu, W.-T., \& Schoelles, M. J. (2006). The soft constraints hypothesis: A rational analysis approach to resource allocation for interactive behaviour. Psychological Review, 113, 461-482.

Griffon-Fouco, M., \& Ghertman, F. (1984). Recueil de données sur les facteurs humains à l'Electricite de France. Operational safety of nuclear power plants (pp. 157-172). Vienna: International Atomic Energy Agency.

Hernstein, R. J., \& Vaughn, W. (1980). Melioration and behavioral allocation. In J. E. R. Stadden (Ed.), Limits to action: The allocation of behavior (pp. 143-176). New York: Academic Press.

Hodgetts, H. M., \& Jones, D. M. (2003). Interruptions in the Tower of London task: Can preparation minimise disruption? Proceedings of the 47th annual meeting of the Human Factors and Ergonomics Society (pp. 1000-1004). Santa Monica: HFES 03.

Hodgetts, H. M., \& Jones, D. M. (2006a). Contextual cues aid recovery from interruption: The role of associative activation. Journal of Experimental Psychology: Learning, Memory, and Cognition, 32, 1120-1132.

Hodgetts, H. M., \& Jones, D. M. (2006b). Interruption of the Tower of London task: Support for a goal-activation approach. Journal of Experimental Psychology: General, 135, 103-115.

Jackson, T. W., Dawson, R., \& Wilson, D. (2002). Evaluating the effect of email interruptions within the workplace. Conference on empirical assessment in software engineering, 3-7.

Jackson, T. W., Dawson, R., \& Wilson, D. (2003). Understanding email interaction increases organizational productivity. Communications of ACM, 46, 80-84. 
Katz, R. (1995). Automatic versus user controlled methods of briefly interrupting telephone calls. Human Factors, 37, 321-334.

Kirlik, A. (1993). Modeling strategic behavior in human-automation interaction - why an aid can (and should) go unused. Human Factors, 35, 221-242.

Latorella, K. A. (1996). Investigating interruptions: Implications for flightdeck performance. Unpublished doctoral dissertation. State University of New York at Buffalo.

Latorella, K. A. (1998). Effects of modality on interrupted flight deck performance: Implications for data link. Proceedings of the 42nd annual meeting of the Human Factors and Ergonomics Society (pp. 249-253). Santa Monica, CA: HFES 98.

Logie, R. H. (1995). Visuo-spatial working memory. Hove: Erlbaum.

Loukopoulos, L. D., Dismukes, R. K., \& Barshi, I. (2001). Cockpit interruptions and distractions: A line observation study. In R. Jensen (Ed.), Proceedings of the 11th international symposium on aviation psychology. Columbus: Ohio State University Press.

Loukopoulos, L. D., Dismukes, R. K., \& Barshi, I. (2003). Concurrent task demands in the cockpit: Challenges and vulnerabilites in routine flight operations. Proceedings of the 12th international symposium on aviation psychology (pp. 737-742). The Wright State University: Dayton.

Lowenstein, G., Read, D., \& Baumeister, R. (2003). Time and decision: Economic and psychological perspectives on intertemporal choice. New York: Russell Sage Foundation.

MacKenzie, I. S. (1992). Fitts' law as a research and design tool in human-computer interaction. Human-Computer Interaction, 7, 91-139.

McDaniel, M. A., Einstein, G. O., Graham, T., \& Rall, E. (2004). Delaying execution of intentions: Overcoming the cost of interruptions. Applied Cognitive Psychology, 18, 533-547.

McFarlane, D. C. (2002). Comparison of four primary methods for coordinating the interruption of people in human-computer interaction. Human-Computer Interaction, 17, 63-139.

McFarlane, D. C., \& Latorella, K. A. (2002). The scope and importance of human interruption in human-computer interaction design. HumanComputer Interaction, 17, 1-61.

Milewski, A. E. (2006). Interruption management and telephone call screening. International Journal of Human-Computer Interaction, 20, 19-33.

Monk, C. A. (2004). The effect of frequent versus infrequent interruption on primary task resumption. Proceedings of the 48th annual meeting of the Human Factors \& Ergonomics Society (pp. 295-299). Santa Monica, CA: HFES 04.

Monk, C. A., Boehm-Davis, D. A., \& Trafton, J. G. (2002). The attentional costs of interrupting task performance at various stages. Proceedings of the 46th annual meeting of the Human Factors and Ergonomics Society (pp. 1824-1828). Santa Monica, CA: HFES 02.

Monk, C. A., Trafton, J. G., \& Boehm-Davis, D. A. (2008). The effect of interruption duration and demand on resuming suspended goals. Journal of Experimental Psychology: Applied, 14, 299-313.

NTSB. (1988, August 16th). Aircraft accident report: Northwest Airlines Inc., McDonnell Douglas DC-9-82, N312RC, Detroit Metropolitan Wayne Country Airport, Romulus, MI, August 16th 1987 (NTSB/AAR88/05). Washington, DC: National Transportation Safety Board.

O'Conaill, B., \& Frohlich, D. (1995). Timespace in the workplace: Dealing with interruptions. Companion proceedings of CHI'95 (pp. 262-263). New York: ACM SIGCHI.

Osgood, C. E. (1949). The similarity paradox in human learning: A resolution. Psychological Review, 56, 132-143.
Paquiot, J., Eyrolle, H., \& Cellier, J. (1986). Conséquences de l'interférence entre les tâches de saisie sur la durée des traitements et l'occurrence des erreurs. In R. Patesson (Ed.), L'homme et L'écran (pp. 199-209). Editions de l'Université de Bruxelles.

Parasuraman, R., \& Riley, V. (1997). Humans and automation: Use, misuse, disuse, abuse. Human Factors, 39, 230-253.

Peterson, L. R., \& Peterson, M. J. (1959). Short-term retention of individual verbal items. Journal of Experimental Psychology, 58, 193-198.

Poulton, E. C. (1982). Influential companions: Effects of one strategy on another in the within-subjects designs of cognitive psychology. Psychological Bulletin, 9, 673-690.

Raaijmakers, J. G. W., \& Shiffrin, R. M. (1980). SAM: A theory of probabilistic search of associative memory. In G. H. Bower (Ed.), The psychology of learning and motivation, Vol. 14 (pp. 207-262). New York: Academic Press.

Rasmussen, J., \& Vicente, K. J. (1989). Coping with human errors through system design: Implications for ecological interface design. International Journal of Man-Machine Studies, 31, 517-534.

Russell, E., Purvis, L. M., \& Banks, A. (2007). Describing the strategies used for dealing with email interruptions according to different situational parameters. Computers in Human Behavior, 23, 1820-1837.

Speier, C., Valacich, J. S., \& Vessey, I. (1999). The influence of task interruption on individual decision making: An information overload perspective. Decision Sciences, 30, 337-360.

Tabachnick, B. G., \& Fidell, L. S. (2001). Using multivariate statistics (4th ed.). Boston: Allyn \& Bacon.

Thorndike, E. L., \& Woodworth, R. S. (1901). The influence of improvement in one mental function upon the efficiency of other functions. Psychological Review, 8, 247-261, 384-, 395, 553-564.

Trafton, J. G., Altmann, E. M., \& Brock, D. P. (2005). Huh, what was I doing? How people use environmental cues after an interruption. Proceedings of the 49th annual meeting of the Human Factors \& Ergonomics Society (pp. 468-472). Santa Monica: HFES 05.

Trafton, J. G., Altmann, E. M., Brock, D. P., \& Mintz, F. E. (2003). Preparing to resume an interrupted task: Effects of prospective goal encoding and retrospective rehearsal. International Journal of HumanComputer Studies, 58, 583-603.

Tucker, A. L., \& Spear, S. J. (2006). Operational failures and interruptions in hospital nursing. Health Services Research, 41, 643-662.

Vicente, K. J., \& Rasmussen, J. (1992). Ecological interface design: Theoretical foundations. IEEE Transactions on systems, man, and cybernetics (pp. 589-606). SMC-22.

Waldron, S. M., Patrick, J., Duggan, G. B., Banbury, S., \& Howes, A. (2008). Designing information fusion for the encoding of visual-spatial information. Ergonomics, 51, 775-797.

Waldron, S. M., Patrick, J., Morgan, P. L., \& King, S. L. (2007). Influencing cognitive strategy by manipulating information access costs. The Computer Journal, 50, 694-702.

Wilson, M. (2002). Six views of embodied cognition. Psychonomic Bulletin \& Review, 9, 625-636.

Woods, D. D., Wise, J., \& Hanes, L. (1981). An evaluation of nuclear power plant safety parameter display systems. Proceedings of the $\mathrm{Hu}$ man Factors 25th Annual Meeting (pp. 110-114). Santa Monica, CA: HFES 81

Zijlstra, F. R. H., Roe, R. A., Leonora, A. B., \& Krediet, I. (1999). Temporal factors in mental work: Effects of interrupted activities. Journal of Occupational and Organisational Psychology, 72, 163-185. 
Appendix

\section{Method for Calculating Movement Time}

Mouse movement times between the target window and both the workspace and resources windows were calculated using a variation of Fitts' law (MacKenzie, 1992). This is a universally accepted approximation of human movement in human-computer interaction and was also used by Gray et al. (2006) in calculating movement time for the BWT. The Fitts' law equation used was $M T=a+b \log _{2}(A / W+1)$, where $M T$ is movement time, $A$ is amplitude (or movement distance) and $W$ is the tolerance or width of the target area. We used the ACT-R parameters for Fitts' law ( $a=0.05 ; b=0.10)$ derived by Card, English, and Burr (1978) that are widely accepted as providing a good fit to moving a mouse cursor, and also used by Gray et al. (2006). The estimated time to make a mouse movement between the target and workspace window in either direction was $196 \mathrm{~ms}$. The estimated times to move from the target to the resource window and vice versa were $265 \mathrm{~ms}$ and $195 \mathrm{~ms}$ respectively.

Received May 8, 2008 Revision received September 14, 2009 Accepted September 21, 2009

\section{Call for Nominations}

The Publications and Communications (P\&C) Board of the American Psychological Association has opened nominations for the editorships of Experimental and Clinical Psychopharmacology, Journal of Abnormal Psychology, Journal of Comparative Psychology, Journal of Counseling Psychology, Journal of Experimental Psychology: General, Journal of Experimental Psychology: Human Perception and Performance, Journal of Personality and Social Psychology: Attitudes and Social Cognition, PsycCRITIQUES, and Rehabilitation Psychology for the years 2012-2017. Nancy K. Mello, PhD, David Watson, PhD, Gordon M. Burghardt, PhD, Brent S. Mallinckrodt, PhD, Fernanda Ferreira, PhD, Glyn W. Humphreys, PhD, Charles M. Judd, PhD, Danny Wedding, $\mathrm{PhD}$, and Timothy R. Elliott, $\mathrm{PhD}$, respectively, are the incumbent editors.

Candidates should be members of APA and should be available to start receiving manuscripts in early 2011 to prepare for issues published in 2012. Please note that the P\&C Board encourages participation by members of underrepresented groups in the publication process and would particularly welcome such nominees. Self-nominations are also encouraged.

Search chairs have been appointed as follows:

- Experimental and Clinical Psychopharmacology, William Howell, PhD

- Journal of Abnormal Psychology, Norman Abeles, PhD

- Journal of Comparative Psychology, John Disterhoft, PhD

- Journal of Counseling Psychology, Neil Schmitt, PhD

- Journal of Experimental Psychology: General, Peter Ornstein, PhD

- Journal of Experimental Psychology: Human Perception and Performance, Leah Light, $\mathrm{PhD}$

- Journal of Personality and Social Psychology: Attitudes and Social Cognition, Jennifer Crocker, PhD

- PsycCRITIQUES, Valerie Reyna, PhD

- Rehabilitation Psychology, Bob Frank, PhD

Candidates should be nominated by accessing APA's EditorQuest site on the Web. Using your Web browser, go to http://editorquest.apa.org. On the Home menu on the left, find "Guests." Next, click on the link "Submit a Nomination," enter your nominee's information, and click "Submit."

Prepared statements of one page or less in support of a nominee can also be submitted by e-mail to Emnet Tesfaye, P\&C Board Search Liaison, at emnet@apa.org.

Deadline for accepting nominations is January 10, 2010, when reviews will begin. 